\title{
Solution of Nonlinear Coupled Heat and Moisture Transport Using Finite Element Method
}

\author{
T. Krejčí
}

This paper deals with a numerical solution of coupled of heat and moisture transfer using the finite element method. The mathematical model consists of balance equations of mass, energy and linear momentum and of the appropriate constitutive equations. The chosen macroscopic field variables are temperature, capillary pressures, gas pressure and displacement. In contrast with pure mechanical problems, there are several difficulties which require special attention. Systems of algebraic equations arising from coupled problems are generally nonlinear, and the matrices of such systems are nonsymmetric and indefinite. The first experiences of solving complicated coupled problems are mentioned in this paper.

Keywords: Coupled hydro-thermo-mechanical analysis, concrete, non-linear system of equations.

\section{Introduction}

Assessment of the durability and serviceability of nuclear power plants is a topical problem in many countries. The most critical part of a power plant is the reactor containment, which is made from concrete with or without a steel lining. There are several requirements on the reactor containment. Basically, it must protect the reactor from external effects, and also the external environment from possible accidents. Mechanical analysis is used for assessing the limit state, and transport analysis is used for describing the leakage of pollutants to the external environment. Possible accidents can lead to great pressure inside the reactor containment, which can cause damage to the concrete, and therefore there is an impact on the transport processes of the pollutants. Therefore coupled hydro-thermo-mechanical analysis is required for a correct assessment of reactor containment properties.

Concrete is a heterogeneous and porous material, which leads to relatively complicated material models. The aim of the present study is to show in a condensed form the theoretical basis of the most widely used mathematical models describing the coupled heat and moisture transport in deforming porous media, to provide a set of governing equations together with the finite element method. The theory discussed below is based on porous media theories given in [1].

\section{Mass and heat transfer in deforming porous media - a review of theory}

\subsection{Constitutive relations}

Moisture in materials can be present as moist air, water and ice or in some intermediate state as an adsorbed phase on the pore walls. Since it is in general not possible to distinguish the different aggregate states, water content $w$ is defined as the ratio of the total moisture weight $(\mathrm{kg} / \mathrm{kg})$ to the dry weight of the material. The degree of saturation $S_{\mathrm{w}}$ is a function of capillary pressure $p^{\mathrm{C}}$ and temperature $T$, which is determined experimentally

$$
S_{\mathrm{w}}=S_{\mathrm{w}}\left(p^{\mathrm{c}}, T\right) \text {. }
$$

The capillary pressure $p^{\mathrm{c}}$ is defined as

$$
p^{\mathrm{c}}=p^{\mathrm{g}}-p^{\mathrm{w}}
$$

where $p^{\mathrm{w}}>0$ is the pressure of the liquid phase (water).

The pressure of the moist air, $p^{\mathrm{g}}>0$, in the pore system is usually considered as the pressure in a perfect mixture of two ideal gases - dry air, $p^{\mathrm{ga}}$, and water vapor, $p^{\mathrm{gw}}$, i.e.,

$p^{\mathrm{g}}=p^{\mathrm{ga}}+p^{\mathrm{gw}}=\left(\frac{\rho^{\mathrm{ga}}}{M_{\mathrm{a}}}+\frac{\rho^{\mathrm{gw}}}{M_{\mathrm{w}}}\right) T R=\frac{\rho^{\mathrm{g}}}{M_{\mathrm{g}}} T R$.

In this relation $\rho^{\mathrm{ga}}, \rho^{\mathrm{gw}}$ and $\rho^{\mathrm{g}}$ stand for the respective intrinsic phase densities, $T$ is the absolute temperature, and $R$ is the universal gas constant.

Identity (3) defining the molar mass of the moist air, $M_{\mathrm{g}}$, in terms of the molar masses of the individual constituents is known as Dalton's law. The greater the capillary pressure, the smaller is the capillary radius. It is shown thermodynamically that the capillary pressure can be expressed unambiguously by the relative humidity $R H$ using the Kelvin-Laplace law

$$
R H=\frac{p^{\mathrm{gw}}}{p^{\mathrm{gws}}}=\exp \left(\frac{p^{\mathrm{c}} M_{\mathrm{w}}}{\rho^{\mathrm{w}} T R}\right) .
$$

The water vapor saturation pressure, $p^{\mathrm{gws}}$, is a function of the temperature only, and can be expressed by the Clausius-Clapeyron equation

$p^{\mathrm{gws}}(T)=p^{\mathrm{gws}}\left(T_{0}\right)=\exp \left[\frac{M_{\mathrm{w}}}{R}\left(\frac{1}{T}-\frac{1}{T_{0}}\right)\right]$,

where $T_{0}$ is a reference temperature and $h_{\text {vap }}$ is the specific enthalpy of saturation.

Materials having heat capacities is the term deliberately used to emphasize the similarity to the description of moisture retention. It is simply expressed as

$$
H=H(T),
$$

where $H$ is the mass specific enthalpy $\left(\mathrm{J} \cdot \mathrm{kg}^{-1}\right), T$ is the temperature $(\mathrm{K})$.

It is not common to write the enthalpy in an absolute way as is done here. Instead, changes of enthalpy are described in a differential way, which leads to the definition of the specific heat capacity as the slope of the $H$-T curve, i.e. 


$$
C_{p}=\left(\frac{\partial H}{\partial T}\right)_{p=\text { const. }} .
$$

The heat capacity varies insignificantly with temperature. It is customary, however, to correct this term for the presence of the fluid phases and to introduce the effective heat capacity as

$$
\left(\rho C_{p}\right)_{\mathrm{eff}}=\rho_{\mathrm{s}} C_{p \mathrm{~s}}+\rho_{\mathrm{w}} C_{p \mathrm{w}}+\rho_{\mathrm{g}} C_{p \mathrm{~g}} .
$$

\subsection{Transfer equations}

The mass averaged relative velocities, $v^{\alpha}-v^{\mathrm{s}}$, are expressed by the generalized form of Darcy's law [1]

$n S_{\alpha}\left(\boldsymbol{v}^{\alpha}-\boldsymbol{v}^{\mathrm{s}}\right)=\frac{k^{\mathrm{r} \alpha} \mathbf{k}_{\mathrm{sat}}}{\mu^{\alpha}}\left(-\operatorname{grad} p^{\alpha}+\rho^{\alpha} \mathbf{g}\right)$,

where $\alpha=\mathrm{w}$ for the liquid phase and $\alpha=\mathrm{g}$ for the gaseous phase.

Dimensionless relative permeabilities $k^{\mathrm{r} \alpha} \in\langle 0,1\rangle$ are functions of the degree of saturation

$$
k^{\mathrm{r} \alpha}=k^{\mathrm{r} \alpha}\left(S_{\mathrm{w}}\right) \quad\left(\mathrm{m} \cdot \mathrm{s}^{-1}\right) .
$$

In Equation (9), $\mathbf{k}_{\mathrm{sat}}\left(\mathrm{m}^{2}\right)$ is the square $(3 \times 3)$ intrinsic permeability matrix and $\mu^{\alpha}$ is the dynamic viscosity $\left(\mathrm{kg} \cdot \mathrm{m}^{-1} \cdot \mathrm{s}^{-1}\right)$. The intrinsic mass densities $\rho^{\alpha}$ are related to the volume averaged mass densities $\rho_{\alpha}$ through the relation

$$
\rho_{\alpha}=n S_{\alpha} \rho^{\alpha} \text {. }
$$

The relative permeability $k^{\mathrm{rw}}$ goes to zero, when water saturation $S_{\mathrm{w}}$ approaches $S_{\mathrm{irr}}$, which is the limiting value of $S_{\mathbf{w}}$ as the suction stress approaches infinity [2].

The diffusive-dispersive mass flux $\left(\mathrm{kg} \cdot \mathrm{m}^{-2} \cdot \mathrm{s}^{-1}\right)$ of the water vapor $(\mathrm{gw})$ in the gas $(\mathrm{g})$ is the second driving mechanism. It is governed by Fick's law

$\boldsymbol{J}_{\mathrm{g}}^{\mathrm{gw}}=n S_{\mathrm{g}} \rho^{\mathrm{gw}}\left(\boldsymbol{v}^{\mathrm{gw}}-\boldsymbol{v}^{\mathrm{g}}\right)=-\rho^{\mathrm{g}} \boldsymbol{D}_{\mathrm{g}} \operatorname{grad}\left(\frac{\rho^{\mathrm{gw}}}{\rho^{\mathrm{g}}}\right)$,

where $\boldsymbol{D}_{\mathrm{g}}\left(\mathrm{m}^{2} \cdot \mathrm{s}^{-1}\right)$ is the effective dispersion tensor. It can be shown [1] that

$$
\begin{aligned}
J_{\mathrm{g}}^{\mathrm{gw}} & =-\rho^{\mathrm{g}} \frac{M_{\mathrm{a}} M_{\mathrm{w}}}{M_{\mathrm{g}}^{2}} \boldsymbol{D}_{\mathrm{g}} \operatorname{grad}\left(\frac{p^{\mathrm{gw}}}{p^{\mathrm{g}}}\right)= \\
& =\rho^{\mathrm{g}} \frac{M_{\mathrm{a}} M_{\mathrm{w}}}{M_{\mathrm{g}}^{2}} \boldsymbol{D}_{\mathrm{g}} \operatorname{grad}\left(\frac{p^{\mathrm{ga}}}{p^{\mathrm{g}}}\right)=-\boldsymbol{J}_{\mathrm{g}}^{\mathrm{ga}} .
\end{aligned}
$$

Here, $J_{\mathrm{g}}^{\mathrm{ga}}$ is the diffusive-dispersive mass flux of the dry air in the gas.

Conduction of heat in the normal sense comprises radiation as well as convective heat transfer on a microscopic level. The generalized version of Fourier's law is used to describe the conduction heat transfer

$$
\boldsymbol{q}=-\chi_{\mathrm{eff}} \operatorname{grad} T
$$

where $\boldsymbol{q}$ is the heat flux $\left(\mathrm{W} \cdot \mathrm{m}^{-2}\right)$, and $\chi_{\text {eff }}$ is the effective thermal conductivity matrix $\left(\mathrm{W} \cdot \mathrm{m}^{-1} \cdot \mathrm{K}^{-1}\right)$.

The thermal conductivity increases with increasing temperature due to the non-linear behavior of the microscopic radiation, which depends on the difference of temperatures raised to the $4^{\text {th }}$ power. The presence of water also increases the thermal conductivity. A suitable formula reflecting this effect can be found in [1].

\section{Deformation of a solid skeleton}

\subsection{Concept of effective stress}

The stresses in the grains, $\sigma^{\mathrm{s}}$, can be expressed using a standard averaging technique in terms of the stresses in the liquid phase, $\sigma^{\mathrm{w}}$, the stresses in the gas, $\sigma^{\mathrm{g}}$, and the effective stresses between the grains, $\sigma^{\text {ef }}$. The equivalence conditions for the internal stresses and for the total stress $\sigma$ lead to the expression [3].

$$
\boldsymbol{\sigma}=n S_{\mathrm{w}} \boldsymbol{\sigma}^{\mathrm{w}}+n S_{\mathrm{g}} \boldsymbol{\sigma}^{\mathrm{g}}+(1-n) \boldsymbol{\sigma}^{\mathrm{s}}+\Delta \boldsymbol{\tau} .
$$

The assumption that the shear stress in fluids is negligible converts the latter equation into the form

$$
\boldsymbol{\sigma}=\boldsymbol{\sigma}^{\mathrm{ef}}-p^{\mathrm{s}} \boldsymbol{m}
$$

where

$\boldsymbol{\sigma}=\left\{\sigma_{x}, \sigma_{y}, \sigma_{z}, \tau_{y z}, \tau_{z x}, \tau_{x y}\right\}^{T}, \quad \boldsymbol{m}=\{1,1,1,0,0,0\}^{T}$

and

$$
p^{\mathrm{s}}=S_{\mathrm{w}} p^{\mathrm{w}}+S_{\mathrm{g}} p^{\mathrm{g}}
$$

Deformation of a porous skeleton associated with the grain rearrangement can be expressed using the constitutive equation written in the rate form

$$
\dot{\boldsymbol{\sigma}}^{\mathrm{ef}}=\boldsymbol{D}_{\mathrm{sk}}\left(\dot{\varepsilon}-\dot{\varepsilon}_{0}\right) \text {. }
$$

The dots denote differentiation with respect to time, $\boldsymbol{D}_{\mathrm{sk}}=\boldsymbol{D}_{\mathrm{sk}}\left(\dot{\varepsilon}, \sigma^{\mathrm{ef}}, T\right)$ is the tangential matrix of the porous skeleton and $\dot{\varepsilon}_{0}$ represents the strains that are not directly associated with stress changes (e.g., temperature effects, shrinkage, swelling, creep). It also comprises the strains of the bulk material due to changes of the pore pressure

$$
\dot{\varepsilon}_{p}=-m\left(\frac{\dot{p}^{\mathrm{s}}}{3 \mathbf{K}_{\mathrm{s}}}\right)
$$

where $\mathbf{K}_{\mathrm{s}}$ is the bulk modulus of the solid material (matrix).

When admitting only this effect and combining Eqs. (16), (19) and (20), we get

$$
\dot{\boldsymbol{\sigma}}=\dot{\boldsymbol{\sigma}}^{\mathrm{ef}}-\dot{p}^{\mathrm{s}} \boldsymbol{m}=\boldsymbol{D}_{\mathrm{sk}} \dot{\varepsilon}-\alpha \boldsymbol{m} \dot{p}^{\mathrm{s}}=\dot{\boldsymbol{\sigma}}^{\prime \prime}-\alpha \boldsymbol{m} \dot{p}^{\mathrm{s}},
$$

where

$$
\alpha=\frac{1}{3} \boldsymbol{m}^{T}\left(\boldsymbol{I}-\frac{\boldsymbol{D}_{\mathrm{sk}}}{3 \mathbf{K}_{m}}\right) \boldsymbol{m}=\frac{\mathbf{K}_{\mathrm{sk}}}{\mathbf{K}_{\mathrm{s}}}<1,
$$

and $\mathbf{K}_{\mathrm{sk}}=\boldsymbol{m}^{T} \boldsymbol{D}_{\mathrm{sk}} \boldsymbol{m} / 9$ is the bulk modulus of the porous skeleton. For a material without any pores, $\mathbf{K}_{\mathrm{sk}}=\mathbf{K}_{\mathrm{s}}$. For cohesive soils, $\mathbf{K}_{\mathrm{sk}} \ll \mathbf{K}_{\mathrm{s}}$ and $\alpha=1$. The above formulas are also applicable to long-term deformation of rocks, for which $\alpha \leq 0.5$, and this fact strongly affects Equation (21) [4]. 
Changes of the effective stress along with temperature and pore pressure changes produce a change in the solid density $\dot{\rho}_{\mathrm{s}}$. To derive the respective material relation for this quantity, we start from the mass conservation equation for the solid phase. In the second step we introduce the constitutive relationship for the mean effective stress expressed in terms of quantities describing the deformation of the porous skeleton. After some manipulations we arrive at the searched formula

$(1-n) \frac{\dot{\rho}^{\mathrm{s}}}{\rho^{\mathrm{s}}}=(\alpha-n)\left(\frac{\dot{p}^{\mathrm{s}}}{\mathbf{K}_{\mathrm{s}}}-\beta_{\mathrm{s}} \dot{T}\right)+(\alpha-1) \operatorname{div} \boldsymbol{v}^{\mathrm{s}}$,

where $\beta_{\mathrm{s}}$ is the thermal expansion coefficient of the solid phase.

A similar approach applied to the mass conservation equation of the liquid phase leads to the following constitutive equation

$$
\frac{\dot{\rho}^{\mathrm{w}}}{\rho^{\mathrm{w}}}=\frac{\dot{p}^{\mathrm{w}}}{\mathbf{K}_{\mathrm{w}}}-\beta_{\mathrm{w}} \dot{T}
$$

where $\mathbf{K}_{\mathrm{w}}$ is the bulk modulus of water and $\beta_{\mathrm{w}}$ is the thermal expansion coefficient of this phase.

\subsection{Set of governing equations}

The complete set of equations describing the coupled moisture and heat transport in deforming porous media comprises the linear balance (equilibrium) equation formulated for a multi-phase body, the energy balance equation and the continuity equations for the liquid water and gas.

Continuity equation for dry air

$\frac{\partial}{\partial t}\left(\varphi\left(1-S_{\mathrm{W}}\right) \rho^{\mathrm{ga}}\right)+\alpha\left(1-S_{\mathrm{W}}\right) \rho^{\mathrm{ga}} \operatorname{div} \dot{\boldsymbol{u}}-$

$-\operatorname{div}\left(\rho^{\mathrm{ga}} \frac{k^{\mathrm{rg}} \boldsymbol{k}_{\mathrm{sat}}}{\mu^{\mathrm{g}}} \operatorname{grad} p_{\mathrm{g}}\right)+$

$+\operatorname{div}\left(\rho^{\mathrm{g}} \frac{M_{\mathrm{a}} M_{\mathrm{w}}}{M_{\mathrm{g}}^{2}} \boldsymbol{D}_{\mathrm{g}} \operatorname{grad}\left(\frac{p^{\mathrm{gw}}}{p^{\mathrm{g}}}\right)\right)=0$,

where $\dot{\boldsymbol{u}}\left(\dot{\boldsymbol{u}}=\boldsymbol{v}^{\mathrm{s}}\right)$ is the velocity of a solid.

\section{Continuity equation for the water species}

$\frac{\partial}{\partial t}\left(\varphi\left(1-S_{\mathrm{W}}\right) \rho^{\mathrm{gw}}\right)+\alpha\left(1-S_{\mathrm{W}}\right) \rho^{\mathrm{gw}} \operatorname{div} \dot{\boldsymbol{u}}-$

$-\operatorname{div}\left(\rho^{\mathrm{gw}} \frac{k^{\mathrm{rg}} \boldsymbol{k}_{\mathrm{sat}}}{\mu^{\mathrm{g}}} \operatorname{grad} p_{\mathrm{g}}\right)+$

$+\operatorname{div}\left(\rho^{\mathrm{g}} \frac{M_{\mathrm{a}} M_{\mathrm{w}}}{M_{\mathrm{g}}^{2}} \boldsymbol{D}_{\mathrm{g}} \operatorname{grad}\left(\frac{p^{\mathrm{gw}}}{p^{\mathrm{g}}}\right)\right)=$

$=\frac{\partial}{\partial t}\left(\varphi S_{\mathrm{w}} \rho^{\mathrm{w}}\right)+\alpha S_{\mathrm{w}} \rho^{\mathrm{w}} \operatorname{div} \dot{\boldsymbol{u}}-$

$-\operatorname{div}\left(\rho^{\mathrm{w}} \frac{k^{\mathrm{rw}} \boldsymbol{k}_{\mathrm{sat}}}{\mu^{\mathrm{w}}}\left(\operatorname{grad} p_{\mathrm{g}}-\operatorname{grad} p_{\mathrm{c}}-\rho^{\mathrm{w}} \boldsymbol{g}\right)\right)$.

\section{Energy balance equation}

$\left(\rho C_{\mathrm{p}}\right)_{\text {eff }} \frac{\partial T}{\partial t}-\operatorname{div}\left(\lambda_{\text {eff }} \operatorname{grad} T\right)-$

$-\operatorname{div}\left(C_{\mathrm{pw}} \rho^{\mathrm{w}} \frac{k^{\mathrm{rw}} \boldsymbol{k}_{\mathrm{sat}}}{\mu^{\mathrm{w}}}\left(\operatorname{grad} p_{\mathrm{g}}-\operatorname{grad} p_{\mathrm{c}}-\rho^{\mathrm{w}} \boldsymbol{g}\right)+\right.$

$\left.+C_{\mathrm{pg}} \rho^{\mathrm{gw}} \frac{k^{\mathrm{rg}} \boldsymbol{k}_{\mathrm{sat}}}{\mu^{\mathrm{g}}} \operatorname{grad} p_{\mathrm{g}}\right) \operatorname{grad} T=$

$=\Delta h_{\mathrm{vap}}\left[\frac{\partial}{\partial t}\left(\varphi S_{\mathrm{w}} \rho^{\mathrm{w}}\right)+\alpha S_{\mathrm{w}} \rho^{\mathrm{w}} \operatorname{div} \dot{\boldsymbol{u}}-\right.$

$\left.-\operatorname{div}\left(\rho^{\mathrm{w}} \frac{k^{\mathrm{rw}} \boldsymbol{k}_{\mathrm{sat}}}{\mu^{\mathrm{w}}}\left(\operatorname{grad} p_{\mathrm{g}}-\operatorname{grad} p_{\mathrm{c}}-\rho^{\mathrm{w}} \boldsymbol{g}\right)\right)\right]$.

The equilibrium equation (the linear balance equation) must still be introduced to complete a set of governing equations

$$
\operatorname{div}\left(\boldsymbol{\sigma}-\boldsymbol{m}\left(p^{\mathrm{g}}-S_{\mathrm{w}} p^{\mathrm{c}}\right)\right)+\rho \boldsymbol{g}=\mathbf{0}
$$

with the density of the multi-phase medium defined as

$\rho=(1-n) \rho^{\mathrm{s}}+n S_{\mathrm{w}} \rho^{\mathrm{w}}+n S_{\mathrm{g}} \rho^{\mathrm{g}}=\rho_{\mathrm{s}}+\rho_{\mathrm{w}}+\rho_{\mathrm{g}}$.

\section{Initial and boundary conditions}

The initial conditions specify the full fields of gas pressure, capillary or water pressure, temperature and displacement and velocities:

$p^{\mathrm{g}}=p_{0}^{\mathrm{g}}, p^{\mathrm{c}}=p_{0}^{\mathrm{c}}, T=T_{0}, \boldsymbol{u}=\boldsymbol{u}_{0}$, and $\dot{\boldsymbol{u}}=\dot{\boldsymbol{u}}_{0}$, at $t=0$.

The boundary conditions can be imposed values on $\Gamma_{\pi}^{1}$ or fluxes on $\Gamma_{\pi}^{2}$, where the boundary $\Gamma=\Gamma_{\pi}^{1}+\Gamma_{\pi}^{2}$,

$p^{\mathrm{g}}=\bar{p}^{\mathrm{g}}$ on $\Gamma_{\mathrm{g}}^{1}, p^{\mathrm{c}}=\bar{p}^{\mathrm{c}}$ on $\Gamma_{\mathrm{c}}^{1}, T=\bar{T}$ on $\Gamma_{T}^{1}$

and $\boldsymbol{u}=\overline{\boldsymbol{u}}$ on $\Gamma_{u}^{1}$.

The volume averaged flux boundary conditions for water species and dry air conservation equations and energy equation to be imposed at the interface between the porous medium and the surrounding fluid are as follows

$$
\begin{array}{lr}
\left(\rho^{\mathrm{ga}} \boldsymbol{J}^{\mathrm{ga}}-\rho^{\mathrm{g}} \boldsymbol{J}^{\mathrm{gw}}\right) \cdot \boldsymbol{n}=\boldsymbol{q}_{\mathrm{ga}} & \text { on } \Gamma_{\mathrm{g}}^{2} \\
\left(\rho^{\mathrm{gw}} \boldsymbol{J}^{\mathrm{ga}}+\rho^{\mathrm{w}} \boldsymbol{J}^{\mathrm{w}}+\rho^{\mathrm{g}} \boldsymbol{J}^{\mathrm{gw}}\right) \cdot \boldsymbol{n}= & \\
=\beta_{\mathrm{c}}\left(\rho^{\mathrm{gw}}-\rho_{\infty}^{\mathrm{gw}}\right)+\boldsymbol{q}_{\mathrm{gw}}+\boldsymbol{q}_{\mathrm{w}} & \text { on } \Gamma_{\mathrm{c}}^{2} \\
-\left(\rho^{\mathrm{w}} \boldsymbol{J}^{\mathrm{w}} \Delta h_{\text {vap }}-\lambda \text { eff } \operatorname{grad} T\right) \cdot \boldsymbol{n}=\alpha_{\mathrm{c}}\left(T-T_{\infty}\right) & \text { on } \Gamma_{T}^{2},
\end{array}
$$

where $\boldsymbol{n}$ is the unit normal vector of the surface of the porous medium, $\rho_{\infty}^{\mathrm{gw}}$ and $T^{\infty}$ are the mass concentration of water vapor and temperature in the undisturbed gas phase far away from the interface, and $\boldsymbol{q}_{\mathrm{ga}}, \boldsymbol{q}_{\mathrm{gw}}, \boldsymbol{q}_{\mathrm{w}}$ and $\boldsymbol{q}_{T}$ are the imposed air flux, the imposed vapor flux, the imposed liquid flux and the imposed heat flux, respectively.

The traction boundary conditions for the displacement field are:

$$
\boldsymbol{\sigma} \cdot \boldsymbol{n}=\boldsymbol{t} \text { on } \Gamma_{u}^{2},
$$

where $\boldsymbol{t}$ is the imposed traction. 


\section{Discretization of governing equations}

A weak formulation of the governing equations (25) to (28) is obtained by applying Galerkin's method of weighted residuals. For the numerical solution, the governing equations are discretized in space by means of the finite element method, yielding a non-symmetric and non-linear system of partial differential equations:

$$
\begin{aligned}
& \mathbf{K}_{\mathrm{uu}} \boldsymbol{u}+\mathbf{K}_{\mathrm{uc}} \boldsymbol{p}_{\mathrm{c}}+\mathbf{K}_{\mathrm{ug}} \boldsymbol{p}_{\mathrm{g}}+\mathbf{K}_{\mathrm{u} t} \boldsymbol{T}=\boldsymbol{F}_{\mathrm{u}}, \\
& \mathbf{C}_{\mathrm{cu}} \dot{\boldsymbol{u}}+\mathbf{C}_{\mathrm{cc}} \dot{\boldsymbol{p}}_{\mathrm{c}}+\mathbf{C}_{\mathrm{cg}} \dot{\boldsymbol{p}}_{\mathrm{g}}+\mathbf{C}_{\mathrm{c} t} \dot{\boldsymbol{T}}+ \\
& +\mathbf{K}_{\mathrm{cu}} \boldsymbol{u}+\mathbf{K}_{\mathrm{cc}} \boldsymbol{p}_{\mathrm{c}}+\mathbf{K}_{\mathrm{cg}} \boldsymbol{p}_{\mathrm{g}}+\mathbf{K}_{\mathrm{ct}} \boldsymbol{T}=\boldsymbol{F}_{\mathrm{c}}, \\
& \mathbf{C}_{\mathrm{gu}} \dot{\boldsymbol{u}}+\mathbf{C}_{\mathrm{gc}} \dot{\boldsymbol{p}}_{\mathrm{c}}+\mathbf{C}_{\mathrm{gg}} \dot{\boldsymbol{p}}_{\mathrm{g}}+\mathbf{C}_{\mathrm{g} t} \dot{\boldsymbol{T}}+ \\
& +\mathbf{K}_{\mathrm{gu}} \boldsymbol{u}+\mathbf{K}_{\mathrm{gc}} \boldsymbol{p}_{\mathrm{c}}+\mathbf{K}_{\mathrm{gg}} \boldsymbol{p}_{\mathrm{g}}+\mathbf{K}_{\mathrm{g} t} \boldsymbol{T}=\boldsymbol{F}_{\mathrm{g}}, \\
& \mathbf{C}_{t \mathrm{u}} \dot{\boldsymbol{u}}+\mathbf{C}_{t \mathrm{c}} \dot{\boldsymbol{p}}_{\mathrm{c}}+\mathbf{C}_{t \mathrm{~g}} \dot{\boldsymbol{p}}_{\mathrm{g}}+\mathbf{C}_{t t} \dot{\boldsymbol{T}}+ \\
& +\mathbf{K}_{t \mathrm{u}} \boldsymbol{u}+\mathbf{K}_{t \mathrm{c}} \boldsymbol{p}_{\mathrm{c}}+\mathbf{K}_{t \mathrm{~g}} \boldsymbol{p}_{\mathrm{g}}+\mathbf{K}_{t t} \boldsymbol{T}=\boldsymbol{F}_{t} .
\end{aligned}
$$

The Eqs. (34) can be rewritten in concise form as

$$
\mathbf{K}(\mathbf{X}) \mathbf{X}+\mathbf{C}(\mathbf{X}) \dot{\mathbf{X}}=\mathbf{F}(\mathbf{X}),
$$

where $\mathbf{X}^{T}=\left\{\boldsymbol{p}^{\mathrm{g}}, \boldsymbol{p}^{\mathrm{c}}, \mathbf{T}, \boldsymbol{u}\right\}, \mathbf{C}(\mathbf{X})$ is "the general capacity matrix", $\boldsymbol{K}(\boldsymbol{X})$ is "the general conductivity matrix" and they are obtained together with $\mathbf{F}(\mathbf{X})$ by assembling the sub-matrices indicated in Eqs. (34). The dot denotes the time derivative.

\section{Method of solution}

Coupled mechanical and transport processes after discretization by the finite element method are described by a system of ordinary differential equations which can be written in the form

$$
\mathbf{K} \boldsymbol{r}+\frac{\mathrm{d} \boldsymbol{r}}{\mathrm{d} t} \mathbf{C}=\boldsymbol{F}
$$

where $\mathbf{K}$ is the stiffness-conductivity matrix, $\mathbf{C}$ is the capacity matrix, $\boldsymbol{r}$ is the vector of nodal values and $\boldsymbol{F}$ is the vector of prescribed forces and fluxes. Numerical solution of the system of ordinary differential Eqs. (36) is based on expressions for unknown values collected in vector $\boldsymbol{r}$ at time $n+1$

$$
\boldsymbol{r}_{n+1}=\boldsymbol{r}_{n}+\Delta t \boldsymbol{v}_{n+\alpha}
$$

where the vector $v_{n+\alpha}$ has the form

$$
\boldsymbol{v}_{n+\alpha}=(1-\alpha) \boldsymbol{v}_{n}+\alpha \boldsymbol{v}_{n+1} .
$$

The vector $v$ contains time derivatives of unknown variables (time derivatives of the vector $\boldsymbol{r}$ ). Eq. (36) is expressed in time $n+1$ and with the help of the previously defined vectors we can find

$(\mathbf{C}+\alpha \Delta t \mathbf{K}) \boldsymbol{v}_{n+1}=\boldsymbol{F}_{n+1}-\mathbf{K}\left(\boldsymbol{r}_{n}+\Delta t(1-\alpha) \boldsymbol{v}_{n}\right)$.

This formulation is suitable because an explicit or implicit computational scheme can be set by parameter $\alpha$. The advantage of the explicit algorithm is based on possible efficient solution of the system of equations, because parameter $\alpha$ can be equal to zero and capacity matrix $\mathbf{C}$ can be diagonal. Therefore the solution of the system is extremely easy. The disadvantage of such a method is its conditional stability. This means that the time step must satisfy the stability condition, which usually leads to a very short time step.
Table 1

\begin{tabular}{|l|l|}
\hline $\begin{array}{l}\text { Initial vectors } \\
\text { (defined by initialconditions) }\end{array}$ & $\boldsymbol{r}_{0}, \boldsymbol{v}_{0}$ \\
\hline do until & $\begin{array}{l}i \leq n \\
(n \text { is number of time steps })\end{array}$ \\
\hline predictor & $\widetilde{\boldsymbol{r}}_{i+1}=\boldsymbol{r}_{i}+(1-\alpha) \Delta t \boldsymbol{v}_{i}$ \\
\hline right hand side vector & $\boldsymbol{y}_{i+1}=\boldsymbol{f}_{i+1}-\mathbf{K} \widetilde{r}_{i+1}$ \\
\hline matrix of the system & $\mathbf{A}=\mathbf{C}+\alpha \Delta t \mathbf{K}$ \\
\hline solution of the system & $\boldsymbol{v}_{i+1}=\mathbf{A}^{-1} \boldsymbol{y}_{i+1}$ \\
\hline new approximation & $\boldsymbol{r}_{i}=\widetilde{\boldsymbol{r}}_{i+1}+\alpha \Delta t \boldsymbol{v}_{i+1}$ \\
\hline
\end{tabular}

The previously described algorithm is valid for linear problems, and one system of linear algebraic equations must be solved in every time step. The situation is more complicated for nonlinear problems, where a nonlinear system of algebraic equations must be solved in every time step. The high complexity of the problems leads to the application of the Newton-Raphson method as the most popular method for such cases.

There are several ways to apply and implement the solver of nonlinear algebraic equations. We prefer equilibrium of forces and fluxes (computed and prescribed) in the nodes. This strategy is based on the equation

$$
f^{\text {int }}+f^{\text {ext }}=0
$$

where vectors $f^{\text {int }}$ and $f^{\text {ext }}$ contain internal values and prescribed values. Due to the nonlinear feature of the material laws used in the analysis, the Eq. (40) is not valid after computation of new values from the equations summarized in Table 1 . There are nonequilibriated forces and fluxes which must be suppressed.

When new values in the nodes are computed, the strains and gradients of the approximated functions can be established. This is done with the help of matrices $\mathbf{B}_{\mathrm{s}}$ and $\mathbf{B}_{\mathrm{g}}$ where particular partial derivatives are collected. Concise expressions of strains and gradients are written as

$$
\boldsymbol{g}=\boldsymbol{B}_{\mathrm{g}} \boldsymbol{r}, \quad \varepsilon=\boldsymbol{B}_{\varepsilon} \boldsymbol{r} .
$$

New stresses and fluxes are obtained from the constitutive relations

$$
\boldsymbol{\sigma}=\boldsymbol{D}_{\sigma} \varepsilon, \quad \boldsymbol{q}=\boldsymbol{D}_{\mathrm{q}} \boldsymbol{g},
$$

where $\boldsymbol{D}_{\sigma}$ is the stiffness matrix of the material and $\boldsymbol{D}_{\mathrm{q}}$ is the matrix of conductivity coefficients. The real nodal forces and the discrete fluxes on an element are computed from the relations

$$
f_{e}^{\mathrm{int}}=\int_{\Omega} \mathbf{B}_{\varepsilon}^{T} \boldsymbol{\sigma} \mathrm{d} \Omega_{e}, \quad f_{e}^{\mathrm{int}}=\int_{\Omega} \mathbf{B}_{\mathrm{g}}^{T} \boldsymbol{q} \mathrm{d} \Omega_{e} .
$$

These are compared with the prescribed nodal forces and discrete fluxes and their differences create the vector of residuals $\boldsymbol{R}$. Increments of vector $v$ are computed from the equation

$$
(\boldsymbol{C}+\alpha \Delta t \boldsymbol{K}) \boldsymbol{v}_{n+1}=\boldsymbol{R}
$$

If matrices $\mathbf{C}$ and $\mathbf{K}$ are updated after every computation of the new increment from Eq. (44), the full Newton-Raphson 


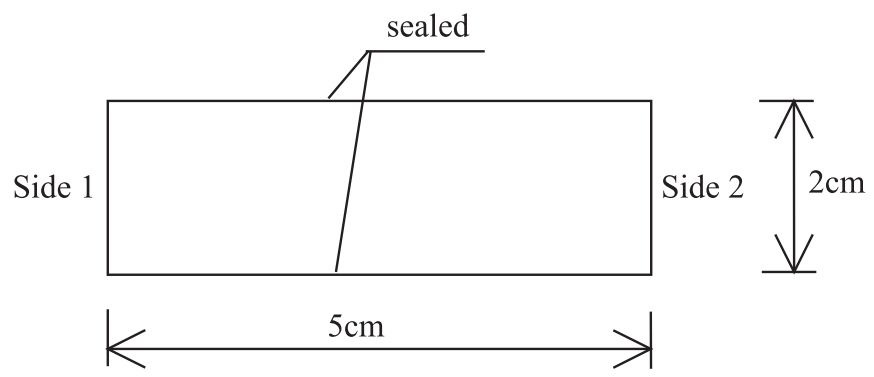

Finite elements: 50 quadrilateral with linear approximation functions

Initial conditions: $\mathrm{T}_{0}=298.15 \mathrm{~K}, p_{\mathrm{c} 0}=9.0 \mathrm{e} 7 \mathrm{~Pa}(50 \% \mathrm{RH}), p_{\mathrm{g} 0}=101325 \mathrm{~Pa}$

Boundary conditions:

$$
\begin{aligned}
& T_{0}-\text { heat flux: } \\
& p^{\mathrm{c}}-\text { Cauchy's } \\
& p^{\mathrm{g}}-\text { Dirichlet's }
\end{aligned}
$$

$\underline{\text { Sidel }}$

$$
\begin{gathered}
q_{T}=1 \mathrm{~K} \cdot \mathrm{min}^{-1}(\text { up to } 475 \mathrm{~K}), \\
\mathrm{RH}=10 \%, \beta \mathrm{c}=0.02 \mathrm{~m} \cdot \mathrm{s}^{-1} ; \\
p^{\mathrm{g}}=101325 \mathrm{~Pa} ;
\end{gathered}
$$

$\underline{\text { Side2 }}$

$$
\begin{gathered}
\alpha \mathrm{c}=20 \mathrm{~W} \cdot \mathrm{m}^{-2} \cdot \mathrm{K}^{-1} \text { (both sides) } \\
\mathrm{RH}=5 \%, \beta \mathrm{c}=0.01 \mathrm{~m} \cdot \mathrm{s}^{-1} \\
p^{\mathrm{g}}=101325 \mathrm{~Pa}
\end{gathered}
$$

Fig. 1: Problem description

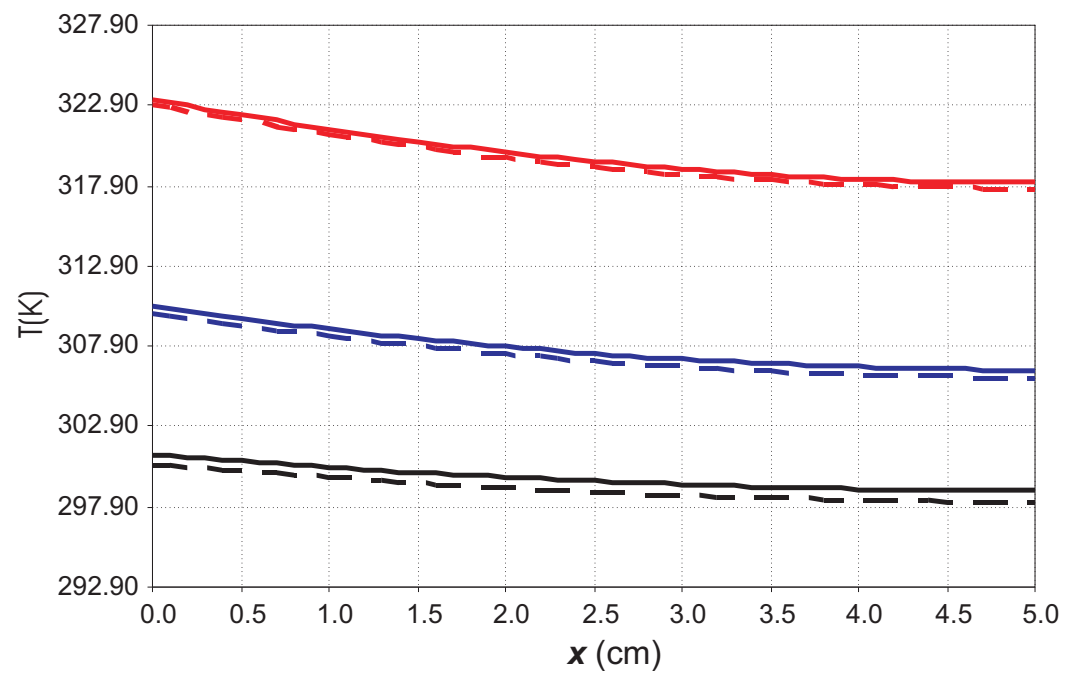

Fig. 2: Temperature profile

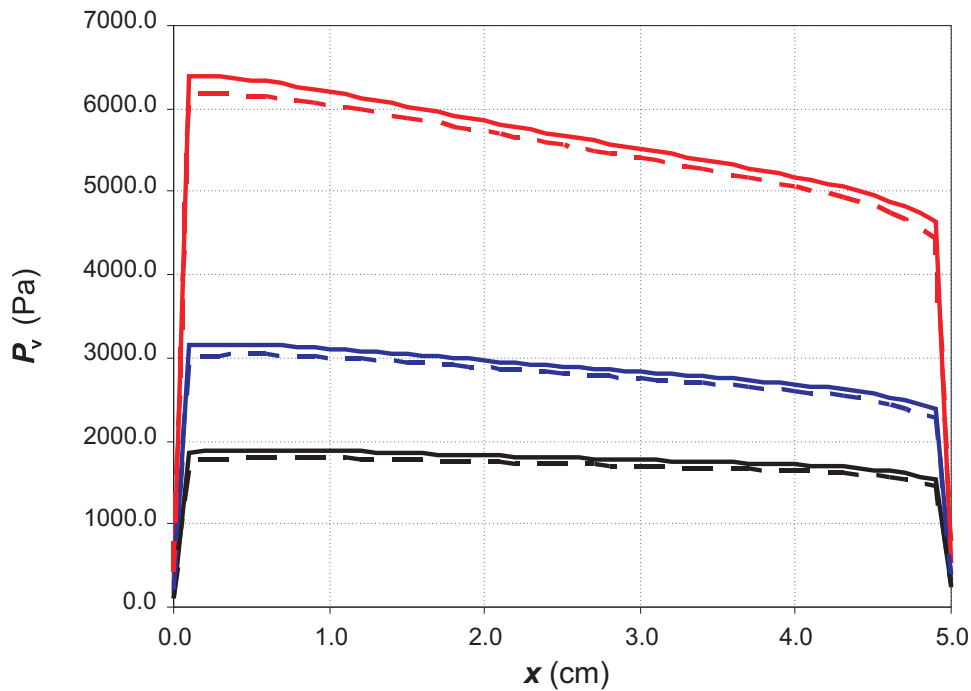

Fig. 3: Water vapour pressure profile 
method is used. If the matrices are updated only once after every time step, the modified Newton-Raphson method is used.

\section{Numerical example}

A simple benchmark test is used to show the differences between a linear and a nonlinear solution. We have a concrete sealed specimen under high temperature conditions, Fig. 1. For this example, concrete is considered as a non-deforming medium (without a displacement field). The material model for ordinary concrete presented by Baroghel-Bouny et al. [8] extended to high temperatures is used. The results are compared after 1, 2 and 4 hours.

\section{Conclusion}

Hydro-thermo-mechanical problems are extremely complicated due to many nonlinearities, and therefore only numerical methods are used for two and three-dimensional problems. A suitable method is the finite element method, where each node has 6 degrees of freedom in three-dimensional problems (3 displacements, temperature and 2 pore pressures). The system of linear algebraic equations (after discretization and linearization) contains many unknowns, and an appropriate solver must be used. Sequential computer codes have severe difficulties with memory and CPU time in connection with such systems. Therefore parallel computers are more often used in complicated analysis. Probably the

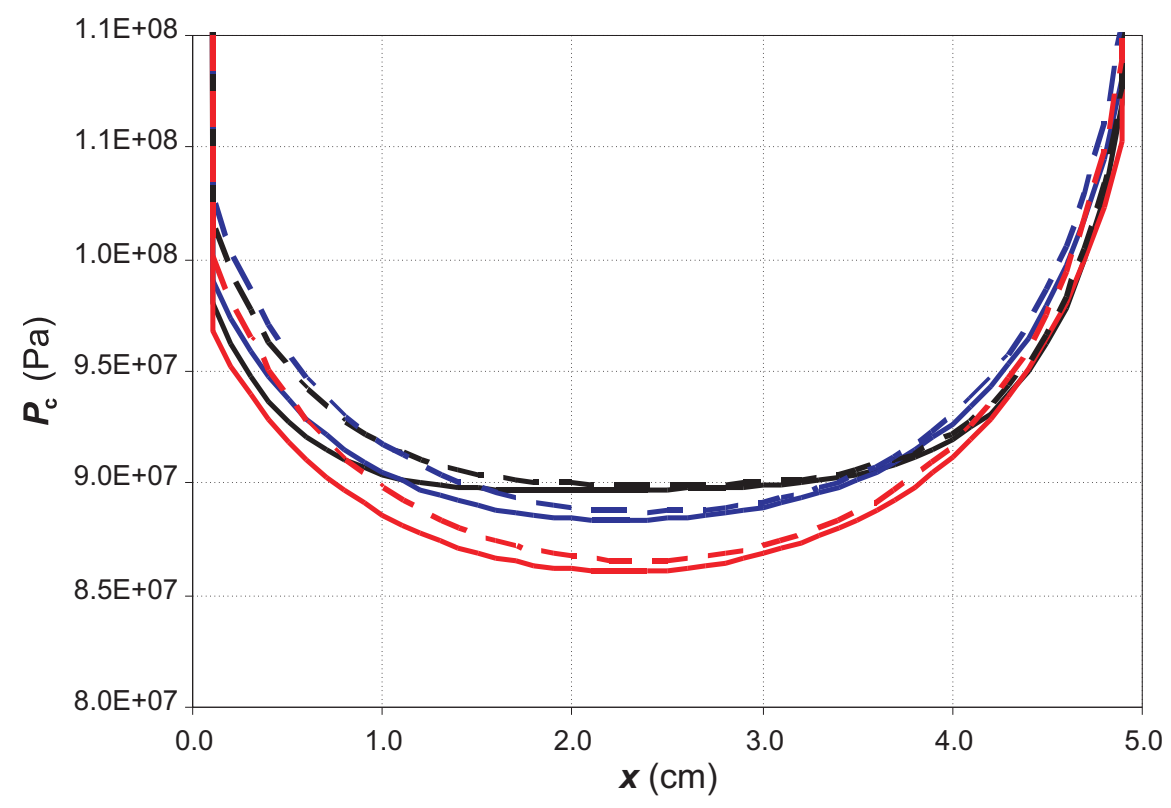

Fig. 4: Capillary pressure profile

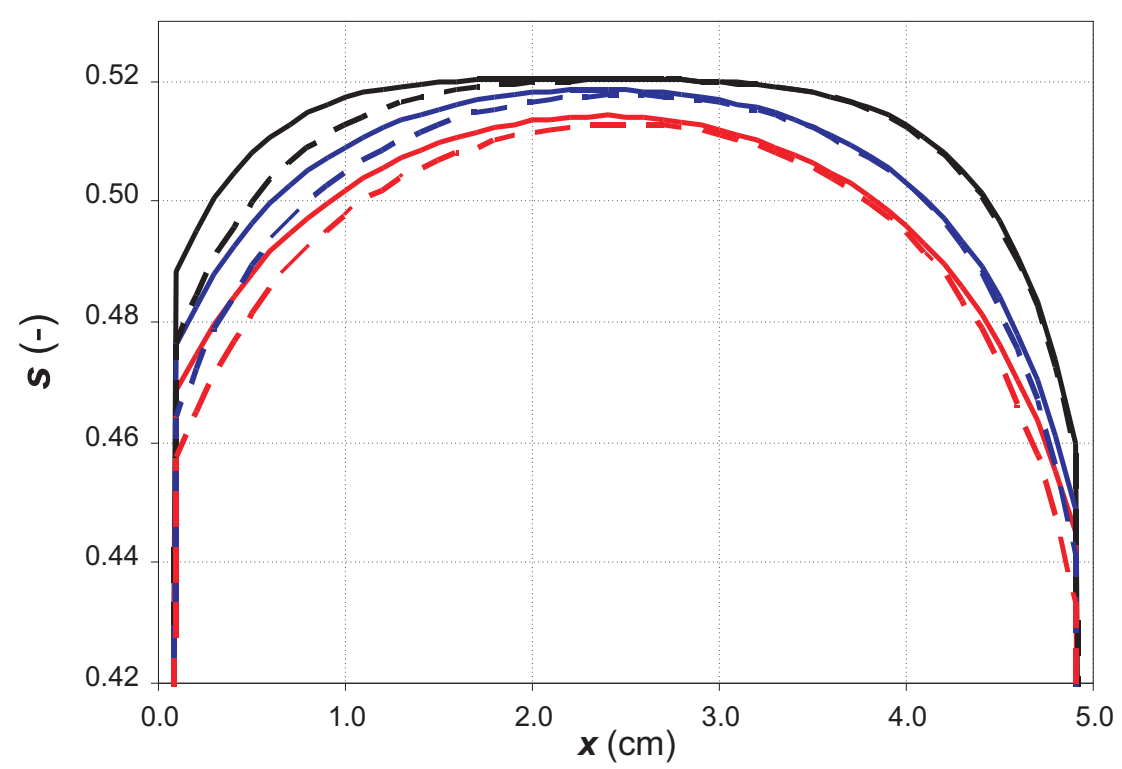

Fig. 5: Degree of saturation profile 
most powerful tool for solving of large systems of equations with the help of parallel computers is the family of domain decomposition methods.

Transport processes lead to nonsymmetric indefinite systems. This means that usual methods, such as $\boldsymbol{L} \boldsymbol{D} \boldsymbol{L}^{\mathrm{T}}$ decomposition, do not work for such problems, and a more general $\boldsymbol{L} \boldsymbol{U}$ decomposition must be used. It seems to us that there are problems where pivoting is necessary.

\section{Acknowledgment}

Financial support for this work was provided by GAČR, contract No. 103/03/D145, and by the EU "Maecenas" project.

\section{References}

[1] Lewis R. W., Schrefler B. A.: "The finite element method in static and dynamic deformation and consolidation of porous media.” John Wiley \& Sons, Chichester-Toronto, 1998, (492).

[2] Fatt I., Klikoff W. A.: "Effect of fractional wettability on multiphase flow through porous media.” Note No. 2043, AIME Trans., 216, 246, 1959.

[3] Bittnar Z., Šejnoha J.: "Numerical methods in structural mechanics.” ASCE Press and Thomas Telford, NY, London, 1996 (422).

[4] Zienkiewicz O. C.: "Basic formulas of static and dynamic behaviour of soils and other porous media." Institute of
Numerical Methods in Engineering. University College of Swansea, 1983.

[5] Krejčí T., Nový T., Sehnoutek L., Šejnoha J.: "Structure Subsoil Interaction in view of Transport Processes in Porous Media." CTU Reports, Vol. 1 (2001), No. 5.

[6] Wang X., Gawin D., Schrefler B. A.: "A parallel algorithm for thermo-hydro-mechanical analysis of deforming porous media." Computational Mechanics 19, Springer-Verlag, 1996, p. 94-104.

[7] Kruis J., Krejčí T., Bittnar Z.: "Numerical Solution of Coupled Problems", in Proceedings of the Ninth International Conference on Civil and Structural Engineering Computing, B. H. V. Topping, (Editor), Civil-Comp Press, Stirling, United Kingdom, paper 24, 2003.

[8] Baroghel-Bouny V., Mainguy M., Lassabatere T., Coussy O.: "Characterization and identification of equilibrium and transfer moisture properties for ordinary and high-performance cementitious materials." Cem. And Conc. Res., Vol. 29 (1999), p.1225-1238.

Ing. Tomáš Krejčí, Ph.D.

phone: +420224354309

e-mail: krejci@cml.fsv.cvut.cz

Department of Structural Mechanics

Czech Technical University in Prague

Faculty of Civil Engineering

Thákurova 7

16629 Prague 6, Czech Republic 